\title{
Photoluminescence Imaging and Wavelength Analysis of Basal Plane Frank-type Defects in 4H-SiC Epilayers
}

\author{
Isaho Kamata, a , Xuan Zhang ${ }^{1, b}$ and Hidekazu Tsuchida ${ }^{1, c}$ \\ ${ }^{1}$ Central Research Institute of Electric Power Industry (CRIEPI), \\ 2-6-1 Nagasaka, Yokosuka, Kanagawa, Japan 240-0196

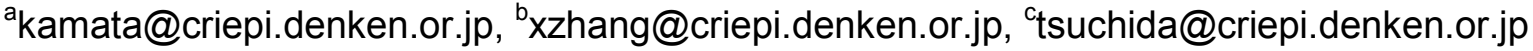

\begin{abstract}
Keywords: Photoluminescence, Photoluminescence imaging microscopy, Frank-type defect
\end{abstract}
\begin{abstract}
Frank-type defects on a basal plane have been investigated using photoluminescence (PL) imaging microscopy and wavelength profile measurement. A wide range of emission in wavelength $(>650 \mathrm{~nm})$ was observed from a Frank partial dislocation at the edge of the defect, while a narrow emission at around the visible light range was obtained from a stacking fault region. The emissions from a stacking fault region of three kinds of basal plane Frank-type defects were confirmed to have different wavelengths depending on their stacking structures.
\end{abstract}

\section{Introduction}

Frank-type defects on a basal plane with four Frank partials, each with a 1/4 $c$ [0001] Burgers vector (multilayer-type defects), can be generated by conversion of a $1 c[0001]$ threading screw dislocation in the substrate during $4 \mathrm{H}-\mathrm{SiC}$ epitaxial growth [1,2]. A long narrow triangle shows stacking fault area and two long edges exhibit the Frank partials in the defect. Extrinsic and intrinsic stacking faults with a 1/4 $c$ [0001] displacement vector (extrinsic- and intrinsic-type defects) are also found in the $4 \mathrm{H}-\mathrm{SiC}$ epilayers. In this study, photoluminescence (PL) imaging microscopy and wavelength profile analysis are performed to investigate PL properties of stacking faults and partials for the three kinds of basal plane Frank-type defects in 4H-SiC epilayers.

\section{Experimental}

4H-SiC epilayers were grown on a commercial $8^{\circ}$ off-cut substrate by vertical hot wall CVD [3]. The samples used for the experiment were thick epilayers exceeding $100 \mu \mathrm{m}$. Using a cooled CCD with a long-pass $(>700 \mathrm{~nm}$ ) filter and band-pass filters for wavelengths of 420, 450 and $480 \mathrm{~nm}$, the PL imaging microscopy was performed at room temperature (RT). An Ar ion laser (364 nm) was used as the excitation source. Wavelength analysis was carried out in two ways. For ultra-violet (UV) to the visible range, a He-Cd laser $(325 \mathrm{~nm})$, monochromator and photomultiplier were used. For the visible to near-infrared range, PL imaging was performed using various band-pass filters for 550, 600, $650,700,742,800,880,900$ and $961 \mathrm{~nm}$. Synchrotron X-ray topography with a monochromatic beam was carried out with 11-2 8 reflection at SPring-8 BL08B2.

\section{Results and discussion}

The PL images obtained with various band-pass filters for the multilayer-type Frank defect are shown in Fig. 1. Figure 1(a) shows a PL image of $420 \mathrm{~nm}$, no obvious emission is observed from the defect region. As shown in Fig. 1(b), a long narrow triangle along the step-flow direction indicating a stacking fault region is observed as a bright contrast in $450 \mathrm{~nm}$, and the region has a dark contrast in 600-900 nm, as shown in Fig. 1(c)-(f). The right side edge of the triangle toward the step-flow direction (the left side in the figures) is observed as a bright contrast in the near-infrared region, as shown in Fig. 1(d)-(f). The edge lines correspond to the Frank partial dislocations on the basal plane. 
(a) 420

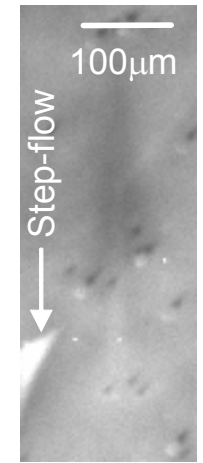

(b) 450

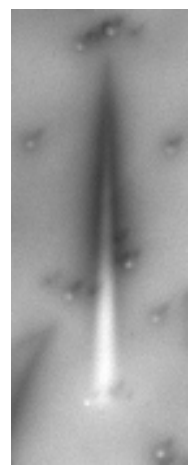

(c) 600

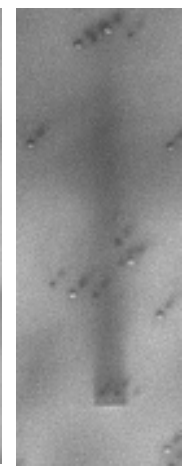

(d) 700

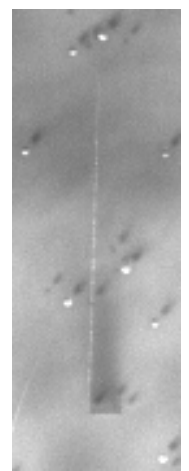

(e) 800

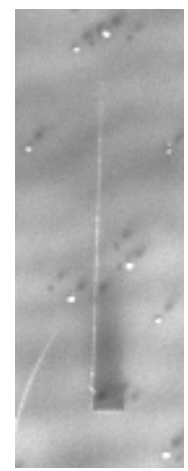

(f) $900 \mathrm{~nm}$

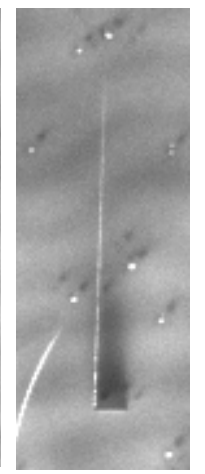

Fig. 1. PL images with band-pass filters for (a) 420, (b) 450, (c) 600, (d) 700, (e) 800 and (f) 900 $\mathrm{nm}$ for the multilayer-type Frank defect.

Figure 2 shows the wavelength profile of the Frank partial dislocation obtained from the PL images at RT with various band-pass filters in the visible to near-infrared range. Wide-ranging wavelength emission and a broad peak at around $750 \mathrm{~nm}$ are clarified for the emission of the Frank partial dislocation.

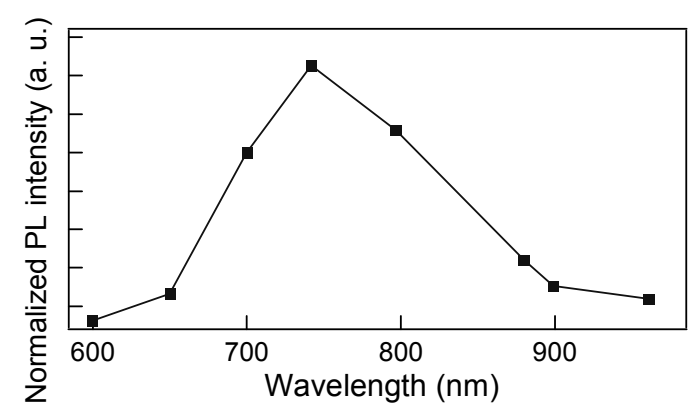

Fig. 2. Wavelength profile of the Frank partial dislocation in the near-infrared range; obtained from PL images with various band-pass filters.

Figure 3(a) shows a PL image of $742 \mathrm{~nm}$ of the multilayer-type Frank defect. Figure 3(b) indicates a line profile of the Frank partial from A' to A along the step-flow direction; corresponding to a line from A' to A in Fig. 3(a). The emission from the partial dislocation at the upstream part exhibited weaker intensity, because the partial dislocation on the basal plane was located at a deeper position from the surface at the upstream part due to the off-cut angle of a substrate.

Figure 3(c) indicates a line profile of the Frank-type defect from B' to B perpendicular to the step-flow direction; corresponding to the line from B' to B shown in Fig. 3(a). The line profile indicates that the stacking fault region shows lower intensity, and the sharp emission is obtained from the edge of the stacking fault region. No strong emission from the other edge is observed in this defect, despite both edges containing partial dislocations.

(a)
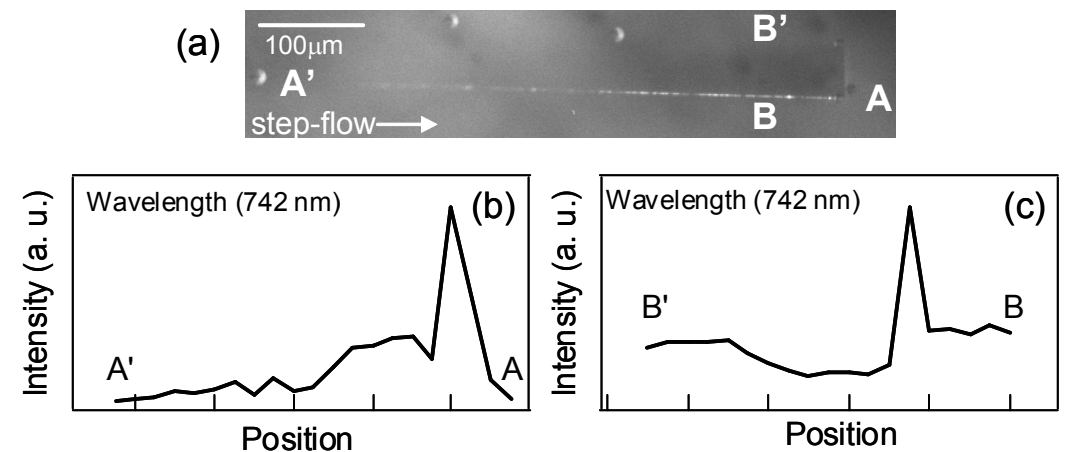

Fig. 3. (a) PL image of $742 \mathrm{~nm}$ and (b) line profiles from A' to A along the step-flow direction and (c) from B' to B perpendicular to the step-flow direction. 

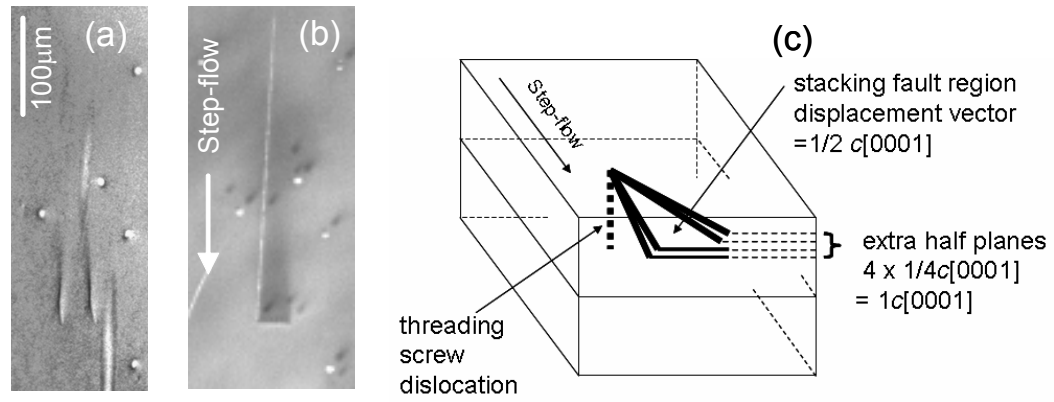

Fig. 4. (a) Synchrotron X-ray topography image for the multilayer-type Frank defect. (b) PL image of a long-pass filter $(>700 \mathrm{~nm})$. (c) Schematic drawing of the defect. The extra half plane generated by the conversion of a $1 c[0001]$ threading screw dislocation in the substrate was terminated by four Frank partials, each with a 1/4 $c[0001]$ at triangle edges.

Figure 4(a) shows the synchrotron X-ray topography image, while Fig. 4(b) indicates the PL image of the long-pass filter $(>700 \mathrm{~nm})$ at the same location with Fig. 1. The edges of the triangle corresponding to the Frank partial dislocations on the basal plane exhibit knife-shaped topography images showing a white long body with a dark edge line at the right side toward the step-flow direction. The dark edge side of the topography image was confirmed to be a terminated side of the extra half plane [1,2], and the stacking fault area with $1 / 2 c[0001]$ displacement vector was clarified to be located between two partial dislocations at the right side and two partials at the left as shown in Fig. 4(c) by high-resolution TEM investigation for this defect. In the case of Fig. 4, the terminated side of the stacking fault is the right side toward the step-flow dislocation, while, a plane of the stacking fault is continuing at the left side. Compared with the X-ray topography and PL images, the edge of the terminated side of the stacking fault is clarified to have a strong emission in the near-infrared range.

Figure 5 shows the wavelength profiles for a stacking fault region of the three kinds of defects at RT. The PL emission profile, peaking at $\sim 457 \mathrm{~nm}$, for a stacking fault of multilayer-type Frank defects was obtained. The extrinsic- and intrinsic-type defects are found to have strong PL emissions at $\sim 424$ and $\sim 488 \mathrm{~nm}$, respectively.

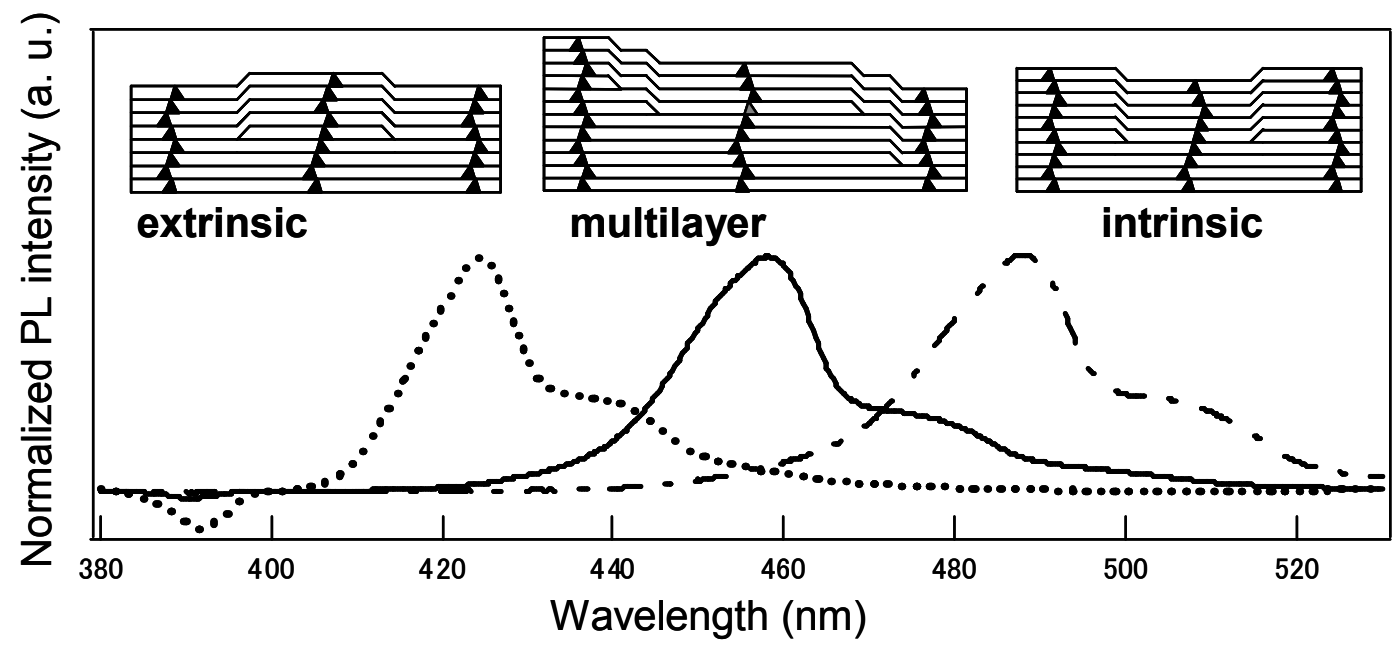

Fig. 5. PL emission profiles for three kinds of Frank-type defects depending on the structure of the stacking fault region.

Stacking sequences were clarified for three types of Frank-type defect by cross-sectional high-resolution TEM and the schematics are shown in Fig. 5. We confirmed the multilayer-type has $(4,2)$ stacking in Zhdanov's notation $[1,2,4]$. The stacking structure of extrinsic and intrinsic 
Frank-type defects was reported as $(4,1)$ and $(5,2)$ [1,2]. In terms of Shockley-type stacking faults or poly-type inclusions, several kinds of structures have been reported as $(3,1)$ for the single Shockley stacking fault, $(4,4)$ for the $8 \mathrm{H}$-stacking fault, $(5,3)$ for the $(3,5)$ type and $(6,2)$ for the double Shockley stacking fault, respectively [5-7].

Figure 6 shows the peak wavelength of the PL emission of the stacking fault area at RT of the Frank- and Shockley-type stacking faults with the stacking notations and schematics. The same schematics were used in the insets of Fig. 5 for three kinds of Frank-type defects. Coincidentally, the peak wavelength of the PL increases as 420, 424, 455, 457, 480, 488 and $500 \mathrm{~nm}$, with increasing the layer number of the $3 \mathrm{C}-\mathrm{SiC}$ potion in their stacking structures.

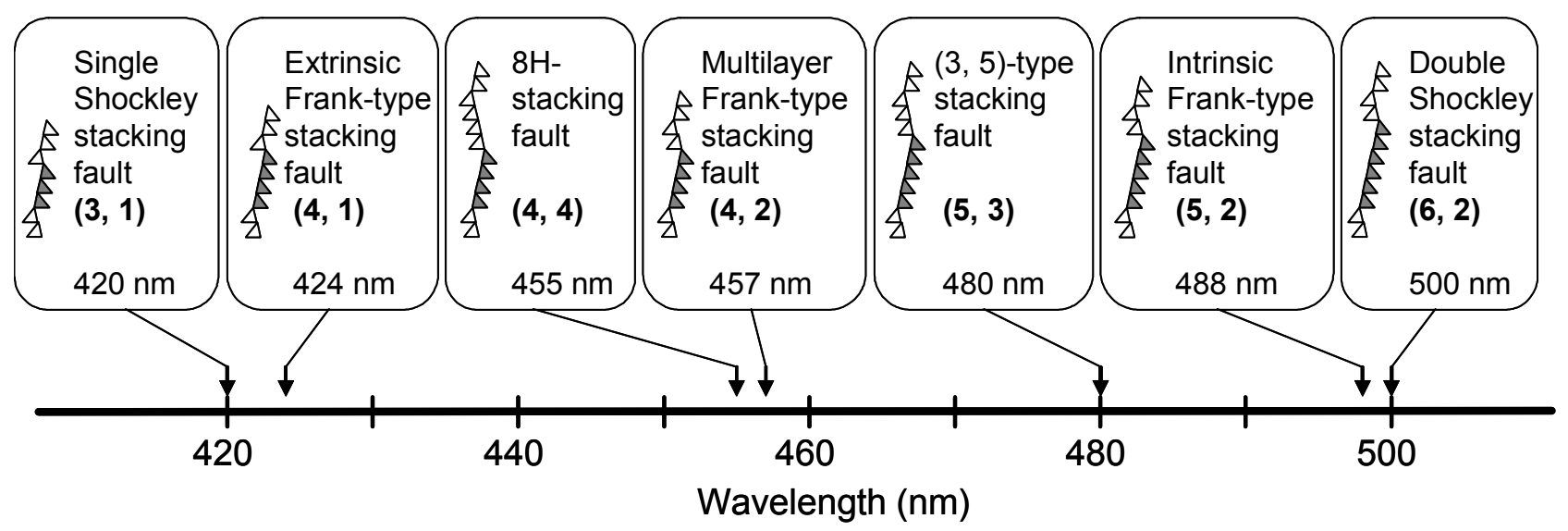

Fig. 6. The PL peak wavelength of Frank- and Shockley-type stacking faults described with stacking notations and schematics.

\section{Summary}

The Frank-type defect was confirmed to have two kinds of luminescence from partial dislocation and the stacking fault region. A wide range of emission in wavelength $(>650 \mathrm{~nm}$ ) was observed from a partial dislocation. Different wavelengths of the PL from the stacking fault region in the visible light range were characterized for three kinds of Frank-type defects; depending on the stacking structures.

\section{Acknowledgments:}

This research is partly supported by the Japan Society for the Promotion of Science (JSPS) through its "Funding Program for World-Leading Innovative R\&D on Science and Technology (FIRST Program)."

\section{References}

[1] H. Tsuchida, I. Kamata, and M. Nagano, J. Crystal Growth 310 (2008) 757.

[2] H. Tsuchida, M. Ito, I. Kamata, and M. Nagano, Phys. Status Solidi B 246, No. 7 (2009) 1553.

[3] H. Tsuchida, I. Kamata, and T. Jikimoto, and K. Izumi, J. Crystal Growth 237-239 (2002) 1206.

[4] I. Kamata, X. Zhang and H. Tsuchida, Appl. Phys. Lett. 97 (2010) 172107.

[5] S. Izumi, H. Tsuchida, I. Kamata, and K. Izumi, Appl. Phys. Lett. 86 (2005) 202108.

[6] G. Feng, J. Suda, and T. Kimoto, Appl. Phys. Lett. 94 (2009) 091910.

[7] G. Feng, J. Suda, and T. Kimoto, Appl. Phys. Lett. 92 (2008) 221906. 